Editorial

\title{
Advancements in Analysis and Design of Protective Structures against Extreme Loadings
}

\author{
Li Chen $\mathbb{D}^{1},{ }^{1}$ Xihong Zhang $\mathbb{D}^{2},{ }^{2}$ Yanchao Shi, ${ }^{3}$ Tuan Ngo, ${ }^{4}$ Chiara Bedon $\left(\mathbb{D},{ }^{5}\right.$ \\ and Piotr W. Sielicki ${ }^{6}$ \\ ${ }^{1}$ Southeast University, Nanjing, China \\ ${ }^{2}$ Curtin University, Perth, Australia \\ ${ }^{3}$ Tianjin University, Tianjin, China \\ ${ }^{4}$ The University of Melbourne, Melbourne, Australia \\ ${ }^{5}$ University of Trieste, Trieste, Italy \\ ${ }^{6}$ Poznan University of Technology, Poznań, Poland \\ Correspondence should be addressed to Li Chen; chenli1360@qq.com
}

Received 25 March 2019; Accepted 25 March 2019; Published 2 May 2019

Copyright ( 2019 Li Chen et al. This is an open access article distributed under the Creative Commons Attribution License, which permits unrestricted use, distribution, and reproduction in any medium, provided the original work is properly cited.

Rising of terrorist bombing threats and industrial explosion accidents highlight the necessity and importance of structural protections against intentional and accidental loadings such as blast and impact. Consequences of such extreme loading conditions have been found to be catastrophic with massive personnel injuries and fatalities, economic loss, and immeasurable social disruption in their wake. It is therefore imperative to enlighten design of modern structures with consideration of protections against such extreme loadings.

Blast and impact loadings have the natures of short duration and high energy intensity, which results in very different structural response as compared to when subjected to conventional quasi-static and less intense dynamic loading such as wind load and seismic load. The analysis and design of conventional structures, therefore, cannot be directly implemented to the design of protective structures against extreme loadings. Moreover, structural response and material behaviour under blast and impact loading are usually nonlinear and time-dependent, involving complex stress states due to stress wave propagation. Simplified analysis and design approaches are not necessarily leading to reliable predictions. Comprehensive understanding about loading characteristics, dynamic material properties, and dynamic response predictions of structures are crucial to reliable and accurate predictions.

Recently, various efforts, including experiment testing, numerical simulation, and analytical derivations, have been devoted to achieving a reliable analysis and design of protective structures. Experimental testing includes both laboratory and field tests, which can demonstrate actual structural behaviours and also be used to validate the fidelity of the numerical model. With the advancement of computer technology and computational mechanics, different numerical methods have been developed and employed to model structural response to blast and impact loadings. Simplified approaches such as single-degree-of-freedom and close-form solutions have been used to predict and analyze structural response. All in all, analysis and design of protective structures against blast and impact loadings, in general, is still a challenge.

This special issue aims to present the state-of-the-art research studies and understandings on the analysis and design of structures against blast and impact loadings as well as other common extreme disaster loadings. Four of the included articles cover an overview of existing blast tests and structural responses under blast loading which include reinforced concrete element, steel concrete structure, and polyurethane foamed aluminum composites. The two articles by P. Y. Yuan et al. and J. Yang et al. discussed response of structures under impact loadings. Moreover, the mechanism of progressive collapse as well as mechanics of other structure responses is also included, which provides fundamental mechanics for the understanding of structural 
behaviour. Last but not least, the understanding of material dynamic properties plays an important role in the analysis of structure response under dynamic loading. Three articles on the study of dynamic material properties including a review of the torsional split Hopkinson pressure bar system are also collected in this special issue, showing complexity of strain rate-dependent materials.

\section{Conflicts of Interest}

There are no conflicts of interest or private agreements.

\section{Acknowledgments}

The papers published have all undergone academic peerreview process. The contribution and support from all the authors and reviewers are to be acknowledged. It is our best wishes that this special issue would serve as a good initiative for more academics to devote on the research of protective structures for the safety of personnel and properties.

$$
\begin{array}{r}
\text { Li Chen } \\
\text { Xihong Zhang } \\
\text { Yanchao Shi } \\
\text { Tuan Ngo } \\
\text { Chiara Bedon } \\
\text { Piotr W. Sielicki }
\end{array}
$$




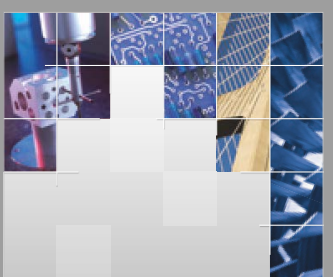

\section{Enfincering}
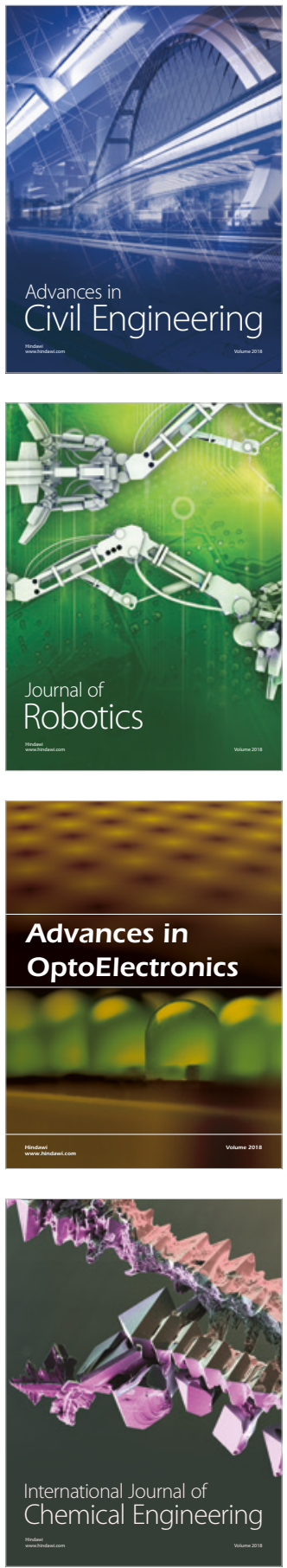

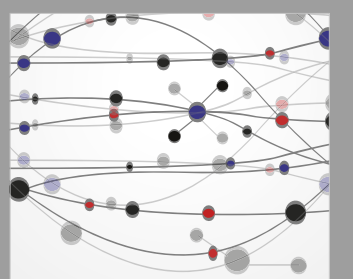

\section{Rotating \\ Machinery}

The Scientific World Journal

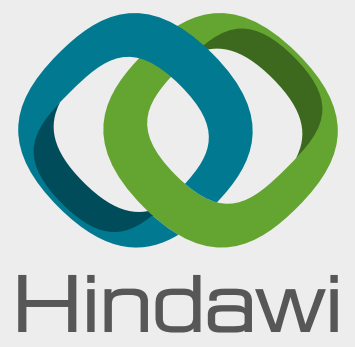

Submit your manuscripts at

www.hindawi.com
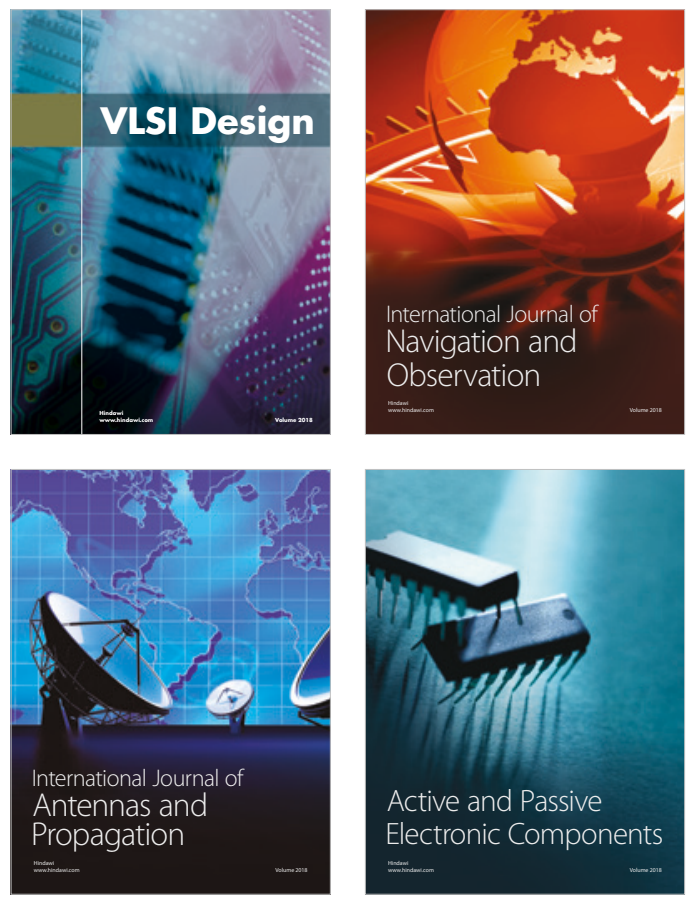
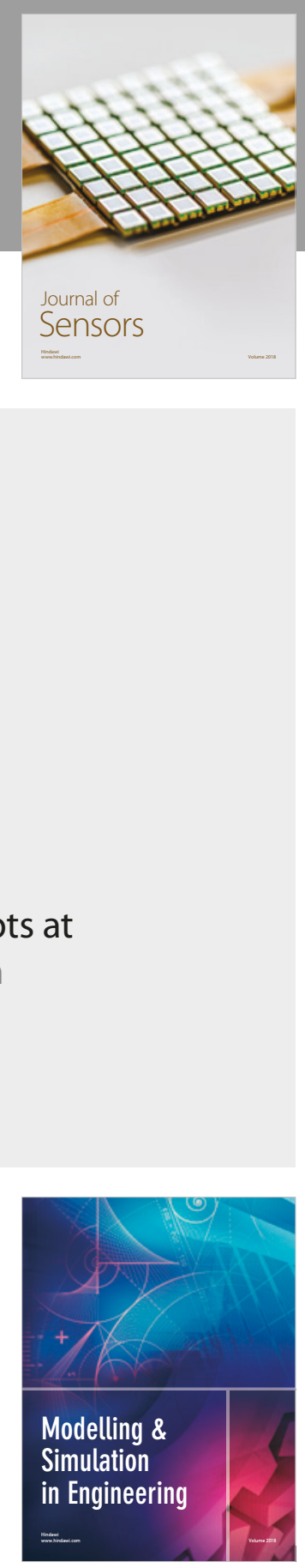

\section{Advances \\ Multimedia}
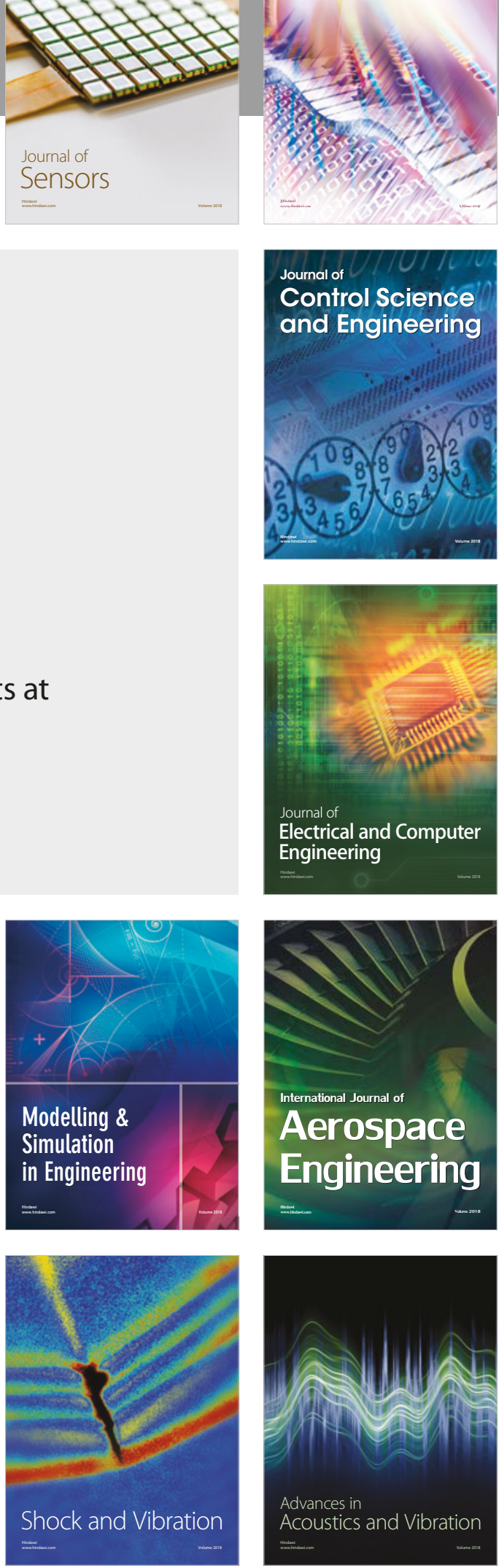